\title{
Bronchopulmonary Dysplasia Associated Pulmonary Hypertension - A Survey of Current Practices in USA and Canada
}

\author{
Amna Qasim*, Aman Jain and Sunil K Jain \\ University of Texas Medical Branch, USA
}

*Corresponding author: Amna Qasim, Department of Pediatrics, University of Texas

Received Date: February 10, 2019

Medical Branch, USA.

Published Date: February 15, 2019

\section{Abstract}

Objective: To evaluate the implementation of the American Heart Association (AHA) and American Thoracic Society (ATS) 2015 guidelines in the diagnosis (including the utility of cardiac catheterization) and management of bronchopulmonary dysplasia associated pulmonary hypertension (BPD-PH) in USA and Canada.

Methods: A validated 25-item questionnaire was sent to academic neonatal centers in USA and Canada to collect information on diagnosis, screening protocols, availability of resources and management of BPD-PH.

Results: 133 responses were included (112 USA and 21 Canadian providers). There were no significant differences in the resources and practices between USA and Canada. ECHO alone is most commonly used to screen infants for BPD-PH and cardiac catheterization is rarely utilized. Treatment of BPD-PH includes use of oxygen to maintain saturations $>95 \%$, Sildenafil, Nitric oxide and combination of all above.

Conclusion: The AHA/ATS guidelines are being implemented partly. There is an urgent need for appropriate and practical guidelines for the management of BPD-PH.

\section{Introduction}

The increased survival of premature infants has led to an increased incidence of complications associated with prematurity. Bronchopulmonary dysplasia (BPD) is the most common chronic lung disease associated with prematurity that affects approximately 10,000-15,000 preterm infants [1] in USA alone with health costs exceeding \$2.4 billion in USA alone [2] Pulmonary hypertension $(\mathrm{PH})$ is one of the most significant complications of BPD due to its high associated morbidity and mortality. $\mathrm{PH}$ complicates approximately one-third infants with BPD cases [3-5] with high mortality (40\%) [6].

There are several risk factors associated with the development of BPD_PH and multiple studies have been done in the past few years to understand this common and significant complication of prematurity. However, due to the scarcity of large-scale studies, questions like when we should screen for BPD_PH, what the appropriate screening tools are and how to optimally manage once screening tests are positive remain unanswered.
The American Heart Association (AHA) and American Thoracic Society (ATS) published the first set of guidelines for BPD-PH in 2015 (summarized in Table 1) [7]. Shortly after that, in 2017, the Pediatric Pulmonary hypertension network published their own guidelines [8]. The purpose of our study was to find out what the current practices are in the management of BPD-PH in USA and Canada in response to the AHA/ATS 2015 guidelines.

Table 1: Summary of AHA/ATS Guidelines for BPD_PH (2015) [7].

1. PH screening by echocardiogram is recommended in infants with established BPD (Class I; Level of Evidence B); Timing of screening:

a) Depending on clinical condition of worsening respiratory distress or risk factors of extreme prematurity (gestational age $<26$ weeks);

b) Every infant at 36 weeks who is diagnosed with moderate or severe BPDi.

2. Evaluation and treatment of lung disease, including assessments for hypoxemia, aspiration, structural airway disease, and the need for changes in respiratory support, are recommended in infants with BPD_ PH before initiation of PAHii-targeted therapy (Class I; Level of Evidence B). 
3. Evaluation for long-term therapy for BPD_PHiii in infants should follow recommendations

for all children with PH and include cardiac catheterization to diagnose disease severity and potential contributing factors such as $\mathrm{LV}$ diastolic dysfunction, anatomic shunts, pulmonary vein stenosis, and systemic collaterals (Class I; Level of Evidence B).

4. Supplemental oxygen therapy is reasonable to avoid episodic or sustained hypoxemia and with the goal of maintaining 02 saturations between $92 \%$ and $95 \%$ in patients with established BPD_PH. (Class IIa; Level of Evidence C).

5. PH-targeted therapy can be useful for infants with BPD_PH on optimal treatment of underlying respiratory and cardiac disease (Class IIa; Level of Evidence C).

6. Treatment with inhaled nitric oxide (iNO) can be effective for infants with established BPD and symptomatic PH (Class IIa; Level of Evidence C).

7. Serial echocardiograms are recommended to monitor the response to PAH-targeted therapy in infants with BPD_PH (Class I; Level of Evidence B).

BPD(i): Bronchopulmonary dysplasia; PAH (ii) Pulmonary arterial hypertension; BPD_PH (iii): BPD associated pulmonary hypertension;

\section{Methodology}

A prospective cross-sectional online survey was conducted from September 2017 to December 2017 in 2 geographically comparable countries: USA and Canada. We chose to look at the practices at academic centers (>Level 3) due to similar state-of-the art health care practices, patient population and availability of resources.

\section{Study objective}

To evaluate the management practices of BPD_PH in the USA and Canada and to determine if the management guidelines recommended by the AHA/ATS in 2015, particularly the utilization of cardiac catheterization in patients with BPD_PH, were being implemented at academic neonatal intensive care units in the USA and Canada.

\section{Inclusion criteria}

Practicing neonatologists, neonatal nurse practitioners and neonatal fellows in the academic neonatal intensive care units in USA and Canada were eligible to participate in this study. We wanted to collect individual responses rather than a single response from one institution since practices may vary widely between providers even if they belong to the same institution.

\section{Survey tool}

A 25-item questionnaire was designed to capture information on resources available at the centers, incidence of BPD and practices related to screening and management of BPD-PH (Figure 1). A survey pilot was conducted on 1 neonatologist, 1 neonatal fellow, 1 pediatric psychologist and 1 pediatric cardiologist to determine content validity, ease of completion and assess the time it took to complete the survey. After incorporating suggestions from the initial responders, the survey was uploaded to an online survey tool (surveymonkey.com). The final questionnaire included questions related to the resources available including availability of cardiac catheterization at the responder's neonatal center, incidence of BPD at their center, management of BPD, screening methods, if any, for BPD-PH and management of BPD-PH. The questions had multiple choice answers and responders were allowed to pick one or more answers as needed. Question 16 asked about screening of BPD-PH, an affirmative response prompted further questions regarding the diagnosis and management of BPD-PH while a negative response resulted in completion of the survey.

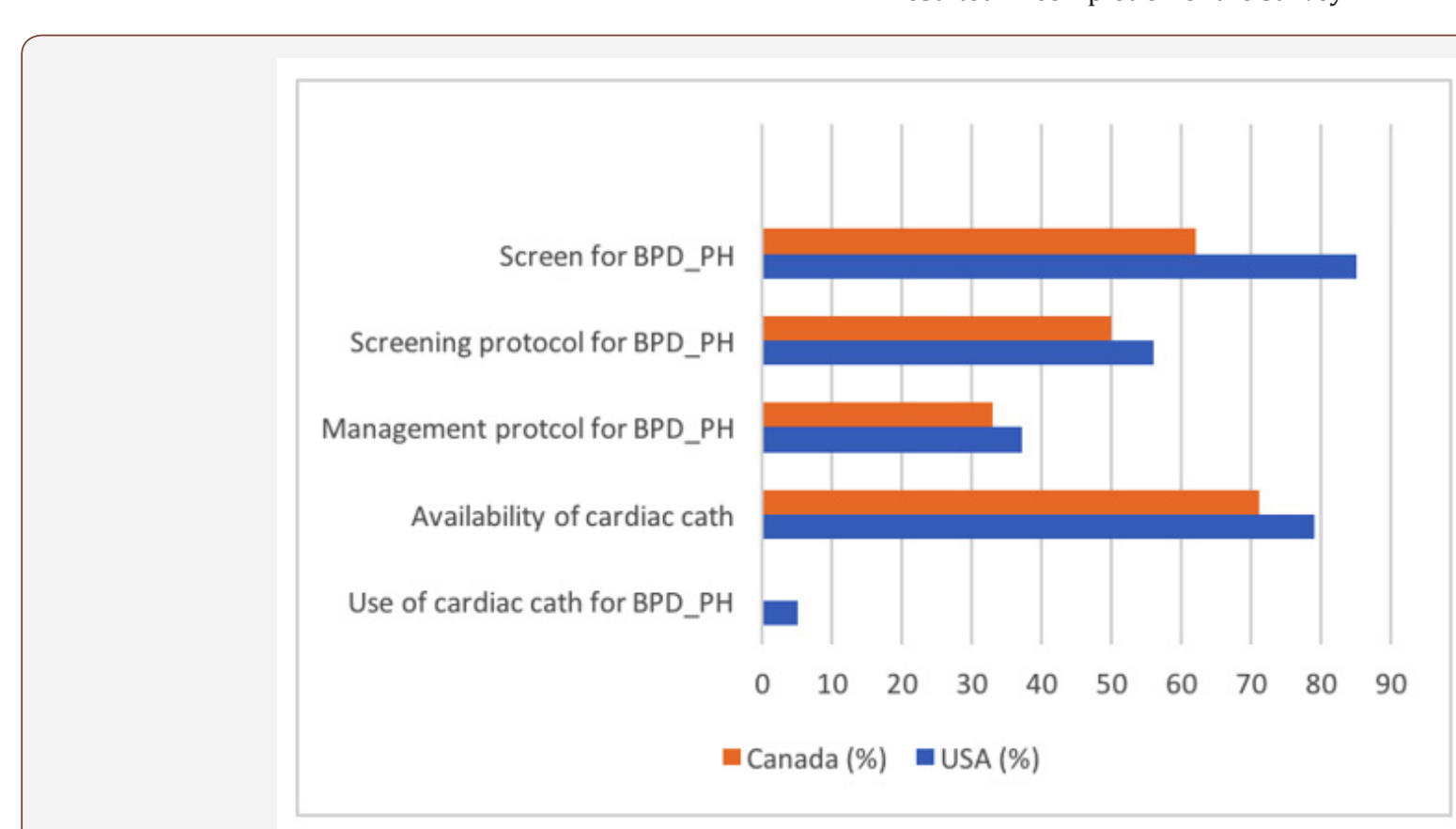

Figure 1: SCREENING AND MANAGEMENT OF BPD_PH: CURRENT PRACTICES IN USA AND CANADA.

\section{Data collection and study end points}

The email addresses of practicing neonatologists in large academic centers in the USA ( $n=99)$ were identified through the
Fellowship and Residency Electronic Interactive Database (FREIDA) and those in Canada $(\mathrm{n}=30)$ were identified through the Canadian Neonatal Network (CNN). An email letter of invitation was sent 
to the neonatal fellowship program directors at these academic centers and they were requested to disseminate the letter amongst neonatologists, neonatal nurse practitioners and neonatal fellows in training at their centers. The survey questionnaire link was embedded in the body of the email invitation letter. Participation in the survey implied consent. Individual identities of the responders were kept confidential throughout data collection, analysis and representation of results. The survey was kept open for 12 weeks and monthly reminders were sent for non-responders. There were no financial or other incentives offered for participation in the survey. Institutional research board approval was obtained from our primary institution.

\section{Sample size and analysis}

Since the goal of this study was to evaluate practices at larger academic centers to see if they were following the latest guidelines, and not to do a specific difference analysis, sample size calculation was not necessary. The responses from all participants were downloaded from the SurveyMonkey database and then exported to SPSS 23 for statistical analysis. Counts with proportions were described for the categorical variables. A comparison between USA and Canada responders was not done due to the small number of responses from Canada.

\section{Results}

Table 2: Demographic characteristics and resources available at centers of responders.

\begin{tabular}{|c|c|}
\hline & n(\%) \\
\hline \multicolumn{2}{|l|}{ Respondents } \\
\hline Neonatal NP & $23(16.5)$ \\
\hline Neonatal Fellow & $34(24.5)$ \\
\hline Neonatologist & $82(59)$ \\
\hline \multicolumn{2}{|l|}{ Number of beds } \\
\hline$<20$ & $6(4.5)$ \\
\hline $21-40$ & $21(16)$ \\
\hline $41-60$ & $38(29)$ \\
\hline$>60$ & $68(51)$ \\
\hline \multicolumn{2}{|l|}{ Population Gestational Age } \\
\hline Any (> 23 weeks) & $131(98.5)$ \\
\hline$>/=28$ weeks & $2(1.5)$ \\
\hline > 30 weeks & \\
\hline Maternal fetal medicine division & $131(98.5)$ \\
\hline Pediatric Surgeon & $131(98.5)$ \\
\hline Pediatric Cardiac Surgeon & $107(80)$ \\
\hline Pediatric Cardiologist & $126(95)$ \\
\hline Pediatric Pulmonologist & $114(86)$ \\
\hline Neonatal Cardiac Catheterization & $103(77)$ \\
\hline
\end{tabular}

A total of 140 neonatal providers (119 from USA and 21 from Canada) responded to the survey. 7 incomplete responses were excluded from final analysis. The exact response rate could not be calculated since we cannot determine how the survey was forwarded by the institutional contacts, but we estimated it to be around $10 \%$. (Table 2) shows the baseline characteristics and resources available at the neonatal intensive care units of the responders. Majority of the responders take care of infants $>23$ weeks' gestational age, have a maternal-fetal medicine division, pediatric surgeons, pediatric cardiologists, pediatric cardiac surgeons, pediatric pulmonologists, extracorporeal membranous oxygenation (ECMO) capabilities and availability of cardiac catheterization.

\section{Practices pertaining to BPD and BPD-PH}

Table 3: Practices related to BPD_PH in USA and Canada.

\begin{tabular}{|c|c|}
\hline & $n(\%)$ \\
\hline \multicolumn{2}{|l|}{ BPD definition } \\
\hline 02 requirement at 36 weeks & $61(46)$ \\
\hline NICHD definition & $68(51)$ \\
\hline Other & $4(3)$ \\
\hline \multicolumn{2}{|l|}{ Overall incidence of BPD in preterm $<32$ weeks } \\
\hline$<10 \%$ & $9(7)$ \\
\hline $11-15 \%$ & $33(34)$ \\
\hline $16-20 \%$ & $30(23)$ \\
\hline$>20 \%$ & $27(20)$ \\
\hline Other & $21(16)$ \\
\hline \multicolumn{2}{|l|}{ Diuretics for BPD } \\
\hline Furosemide & $8(6)$ \\
\hline HCTZ & $8(6)$ \\
\hline HCTZ + Spironolactone & $63(48)$ \\
\hline Other & $51(40)$ \\
\hline Screening for BPD_PH & $106(79)$ \\
\hline Screening Protocol for BPD_PH & $53(54)$ \\
\hline \multicolumn{2}{|l|}{ Screening tools } \\
\hline ECHO & $74(75.5)$ \\
\hline Biomarkers (BNP/NTproBNP) & $2(2)$ \\
\hline ECHO + biomarkers & 17 (17.4) \\
\hline Other & $5(5.1)$ \\
\hline \multicolumn{2}{|l|}{ Time of screening } \\
\hline 32 weeks & $13(14)$ \\
\hline 34 weeks & $4(4)$ \\
\hline 36 weeks & $38(40.4)$ \\
\hline After 36 weeks & 18 (19.1) \\
\hline Other & $21(22.4)$ \\
\hline \multicolumn{2}{|l|}{ ECHO parameters for screening } \\
\hline Quantitative & $8(8.4)$ \\
\hline Qualitative & $7(7.4)$ \\
\hline Both & $80(84.2)$ \\
\hline Confirmation by Cardiac catheterization & $5(5)$ \\
\hline Management protocol for BPD-PH & $36(37)$ \\
\hline
\end{tabular}

(Table 3) shows a summary of the practices pertaining to BPD and BPD_PH. Practices did not differ significantly in the USA and Canada. National Institute of Child Health and Human Development (NICHD) definition [9] of BPD is more commonly used in USA vs Canada (55\% vs 33\%) while oxygen requirement at 36 weeks was used to define BPD in Canada. The physiologic definition of BPD was not commonly used by the responders [10] Incidence of BPD varied depending on the population, with the highest 
incidence reported in preterm infants $<1000$ grams. Management of BPD involved optimization of respiratory support and nutrition. Supportive treatment with diuretics was commonly reported with the most common combination being hydrochlorothiazide and spironolactone ( $45 \%$ vs $67 \%$ ). Most responders admitted that treatment choices depended heavily on a case by case basis and practices varied from provider to provider. The anonymity of our survey did not allow us to look at differences in practices from the same institutes.

\section{Practices pertaining to BPD-PH}

$82 \%$ USA vs $62 \%$ Canadian providers reported screening for BPD_PH and 55\% vs 50\% reported having a screening protocol for BPD_PH. In the USA, ECHO alone (72\%) and ECHO plus biomarkers like BNP/NTproBNP (20\%) are usually used for screening for BPD-PH, while in Canada ECHO alone (100\%) was reported as the screening tool for BPD-PH. Timing of screening varies but the most common time of screening is at 36 weeks' gestational age in both US and Canada. Some responders reported that screening was done according to clinical symptoms at any gestational age. Both quantitative (Tricuspid jet velocity, left ventricular eccentricity index etc.) and qualitative echocardiographic (interventricular septal flattening, right ventricular hypertrophy, dilatation of the right atrium and dilatation of the right ventricle) measures are used for screening for BPD_PH.

Majority of the responders (62\% USA vs 67\% Canadian) reported not having a management protocol for BPD-PH. Management of BPD-PH usually involves a multidisciplinary approach with neonatal intensive care, pediatric cardiology and pediatric pulmonology teams. Treatment usually varies according to severity and typically involves a combination of maintenance of oxygen saturations $>92 \%$, nitric oxide, pulmonary vasodilators like sildenafil and diuretics.

Treatment of BPD-PH includes use of oxygen to maintain saturations > 95\% ( $4 \%$ vs $54 \%$ ), Sildenafil ( $6 \%$ vs $73 \%$ ), Nitric oxide (5\% vs $73 \%)$ and combination of all above ( $63 \%$ vs $18 \%)$.

\section{Utilization of cardiac catheterization in the management of BPD-PH}

Cardiac catheterization was rarely utilized (5\%) to confirm the diagnosis prior to initiation of therapy. The main reasons for not utilizing cardiac catheterization included risks associated with the procedure and/or lack of expertise available to perform the procedure on neonates. A few responders reported utilizing cardiac catheterization in patients that did not respond to pulmonary vasodilators, in cases where pulmonary venous obstruction was suspected or if recommended by cardiology.

\section{Discussion}

$\mathrm{PH}$ is a serious complication of BPD and is an area of growing interest as incidence BPD_PH is increasing with the improved survival of extremely preterm infants. Severe $\mathrm{PH}$ beyond the first few months of life is associated with a $47 \%$ mortality rate within 2 years after diagnosis [11]. This is one of the initial efforts to determine medical practices pertaining to BPD_PH specially utilization of cardiac catheterization in the management of BPD$\mathrm{PH}$ as recommended by AHA/ATS guidelines1. A similar study was recently published (after our survey was completed) that looked at practices of American Academy of Pediatrics neonatology members [12].

The results of our survey show that most neonatal providers recognize BPD-PH as a significant complication and are screening for it. However, only about half of them reported having a screening protocol and less than half have a management protocol. As recommended by the AHA/ATS guidelines, quantitative and qualitative ECHO parameters are used for screening of BPD-PH at most centers. We believe that ECHO is a preferred diagnostic tool may be due non-invasive and ease of availability. Mourani et al showed that ECHO identifies $\mathrm{PH}$ in $79 \%$ of patients under 2 years of age [13]. The most commonly used ECHO parameter to determine $\mathrm{PH}$ is tricuspid jet velocity (TRJV) that is used to calculate the estimated right ventricular systolic pressure via the modified Bernoulli equation. PH is calculated as the sum of right ventricular systolic pressure and right atrial pressure. However, TRJV is measurable in only $31-61 \%$ of infants and absence of TRJV does not rule out PH $[13,14]$ In these cases, other ECHO parameters like right atrial enlargement, right ventricular hypertrophy and/ or dilatation, interventricular septal flattening during systole, left ventricular eccentricity index (LV SEI) and time to peak velocity/ right ventricular ejection time ration (TPV/RVET) may be used $[4,14]$.

Brain natriuretic peptide (BNP) is released by cardiac myocytes in response to stretch of cardiac muscles and is used as an indirect marker of pulmonary hypertension. Studies involving extremely low birth weight infants have correlated high serum BNP levels with increased risk of mortality [15]. N-terminal pro BNP (NTproBNP) is the more stable byproduct of BNP and has a longer half-life. AHA recommends the use of biomarkers BNP and NTproBNP at the time of diagnosis and during follow up to supplement clinical decisions [7]. Our results show that only $22 \%$ of the centers in the USA are using these biomarkers for screening of BPD_PH before starting treatment and none of the Canadian centers reported using these biomarkers.

This study has a lot of limitations. It is important to realize that a survey does not always represent actual clinical practices, especially when the response rate is low. Wide variations exist in practices amongst providers at the same centers and due to the anonymity of our survey, we were not able to tease out these differences or even determine the actual number of neonatal centers represented in our responses. Another major limitation is that only academic neonatal centers were included in our survey - resources will likely differ if all neonatal centers are taken into account and may drastically affect the overall practices pertaining to the screening and management of BPD-PH.

\section{Cardiac catheterization - a futile recommendation}

The AHA/ATS recommends obtaining cardiac catheterization prior to initiation of treatment of BPD-PH. We found that even though cardiac catheterization is available at most of the centers (in almost 3/4th centers of those who responded), it is very rarely 
utilized prior to initiation of therapy in cases of BPD_PH (only 5\% responders from USA reported using it in special circumstances like no response to therapy and suspicion of cardiac shunting lesions). However, in the recent survey conducted by Altit et al, $33 \%$ reported never obtaining a cardiac catheterization, 36\% did so rarely, $23 \%$ reported utilizing it occasionally, $7 \%$ often and $1 \%$ reported utilizing it always prior to initiation of medication.

The use of cardiac catheterization would be very helpful in determining critical information like presence of pulmonary venous obstruction, left ventricular diastolic dysfunction, severity of associated cardiac lesions, identification of collaterals and assessment of pulmonary vascular reactivity. However, practically it is very challenging and difficult to get cardiac catheterization in very sick infants who are suspected to have BPD-PH. In addition to the complications associated with the procedure, especially an increased risk of cardiac arrest \& mortality in pediatric patients with PH [16], lack of technical expertise and presence of other comorbidities limits the utility of this so called "gold standard per AHA recommendations" in preterm infants.

\section{Conclusion}

Practices pertaining to the management of BPD_PH are largely similar at centers in the USA and Canada. The AHA/ATS guidelines of 2015 are only being implemented partly. Cardiac catheterization is very rarely utilized to confirm the diagnosis of BPD-PH prior to initiation of treatment and is likely not a practically applicable recommendation. There is an urgent need for appropriate and practical guidelines for the management of BPD_PH.

\section{Acknowledgement}

None.

\section{Conflict of Interest}

The authors declare no conflict of interest.

\section{References}

1. Van Marter LJ (2009) Epidemiology of bronchopulmonary dysplasia. Seminars in Fetal \& Neonatal Medicine 14(6): 358-366.

2. McAleese KA, Knapp MA, Rhodes TT (1993) Financial and emotional cost of bronchopulmonary dysplasia. Clinical Pediatrics 32(7): 393-400.
3. Bhat R, Salas AA, Foster C, Carlo WA, Ambalavanan N (2012) Prospective analysis of pulmonary hypertension in extremely low birth weight infants. Pediatrics 129(3): 682-689.

4. Kim GB (2010) Pulmonary hypertension in infants with bronchopulmonary dysplasia. Korean journal of pediatrics 53(6): 688693.

5. Al-Ghanem G, Shah P, Thomas S, Banfield L, el Helou S, et al. (2017) Bronchopulmonary dysplasia and pulmonary hypertension: a metaanalysis. J Perinatology 37(4): 414-419.

6. Bui CB, Pang MA, Sehgal A, Theda C, Lao JC, et al. (2017) Pulmonary hypertension associated with bronchopulmonary dysplasia in preterm infants. J Reproductive Immunol 124: 21-29.

7. Abman SH, Hansmann G, Archer SL, Ivy DD, Adatia I, et al. (2015) Pediatric pulmonary hypertension Guidelines from the american heart association and american thoracic society. Circulation 132(21): 20372099.

8. Krishnan U, Feinstein JA, Adatia I, Austin ED, Mullen MP, Hopper RK, et al. (2017) Evaluation and management of pulmonary hypertension in children with bronchopulmonary dysplasia. The J pediatrics. 24-34.

9. Ehrenkranz RA, Walsh MC, Vohr BR, Jobe AH, Wright LL, et al. (2005) Validation of the national institutes of health consensus definition of bronchopulmonary dysplasia. Pediatrics 116(6): 1353-1360.

10. Walsh MC, Yao Q Network NN, Hale E, Collins M, et al. (2003) Impact of prospective application of a physiologic definition of bronchopulmonary dysplasia. Pediatric 53(4): 403-403.

11. Khemani E, McElhinney DB, Rhein L, Andrade O, Lacro RV, et al. (2007) Pulmonary artery hypertension in formerly premature infants with bronchopulmonary dysplasia: Clinical features and outcomes in the surfactant era. Pediatrics 120(6): 1260-1269.

12. Altit G, Lee HC, Hintz S, Tacy TA, Feinstein JA, et al. (2018) Practices surrounding pulmonary hypertension and bronchopulmonary dysplasia amongst neonatologists caring for premature infants. Journal of Perinatology 38(4): 361-367.

13. Mourani PM, Sontag MK, Younoszai A, Ivy DD, Abman SH (2008) Clinical utility of echocardiography for the diagnosis and management of pulmonary vascular disease in young children with chronic lung disease. Pediatrics 121(2): 317-325.

14. Revanna GK, Kunjunju A, Sehgal A (2017) Bronchopulmonary dysplasia associated pulmonary hypertension: Making the best use of bedside echocardiography. Progress in Pediatric Cardiology 46: 39-43.

15. Cuna A, Kandasamy J, Sims B (2014) B-type natriuretic peptide and mortality in extremely low birth weight infants with pulmonary hypertension: a retrospective cohort analysis. Bmc Pediatr 14: 68.

16. Taylor CJ, Derrick G, McEwan A, Haworth SG, Sury MRJ (2007) Risk of cardiac catheterization under anaesthesia in children with pulmonary hypertension. British Journal of Anaesthesia 98(5): 657-661. 